\title{
Utility of ALK-1 protein expression and $A L K$ rearrangements in distinguishing inflammatory myofibroblastic tumor from malignant spindle cell lesions of the urinary bladder
}

\author{
William R Sukov ${ }^{1}$, John C Cheville ${ }^{1, *}$, Austin W Carlson ${ }^{1}$, Brandon M Shearer ${ }^{1}$, \\ Eli J Piatigorsky ${ }^{1}$, Karen L Grogg ${ }^{1}$, Thomas J Sebo ${ }^{1}$, Jason P Sinnwell ${ }^{2}$ and \\ Rhett P Ketterling,*
}

${ }^{1}$ Department of Laboratory Medicine and Pathology, Mayo Clinic, Rochester, MN, USA and ${ }^{2}$ Department of Health Sciences Research, Mayo Clinic, Rochester, MN, USA

\begin{abstract}
Inflammatory myofibroblastic tumor of the urinary bladder is an unusual spindle cell neoplasm that displays cytologic atypia, infiltrative growth and mitotic activity mimicking malignant tumors, such as leiomyosarcoma, rhabdomyosarcoma and sarcomatoid carcinoma. The objective of this study was to determine if anaplastic lymphoma kinase (ALK-1) protein expression detected by immunohistochemistry and ALK rearrangements detected by fluorescence in situ hybridization (FISH) were useful in distinguishing inflammatory myofibroblastic tumor from malignant spindle cell tumors of the urinary bladder. In inflammatory myofibroblastic tumor, ALK-1 expression was identified in 13 of 21 cases $(62 \%)$ and $A L K$ rearrangements in 14 of 21 cases $(67 \%)$. All cases of inflammatory myofibroblastic tumor demonstrating ALK-1 expression, carried $A L K$ rearrangements. One case negative for ALK-1 expression exhibited $A L K$ rearrangement. $A L K$ rearrangements were more common in women $(\boldsymbol{P}=\mathbf{0 . 0 0 3 2})$. Leiomyosarcoma, sarcomatoid carcinoma, embryonal rhabdomyosarcoma and reactive myofibroblastic proliferations were negative for ALK-1 protein and ALK rearrangements. Immunohistochemistry using markers of muscle, epithelial, neural, and follicular dendritic cell differentiation showed overlap between inflammatory myofibroblastic tumor with and without $A L K$ gene rearrangements, and between inflammatory myofibroblastic tumor and spindle cell malignancies. However, coexpression of cytokeratin and muscle-specific antigens was unique to inflammatory myofibroblastic tumor, observed in approximately half the tumors. This study indicates that detection of ALK protein and $A L K$ gene rearrangements are useful in distinguishing inflammatory myofibroblastic tumor from spindle cell malignancies in the urinary bladder. Additionally, our findings suggest that $A L K$ rearrangement is the primary mechanism for $A L K$ activation and that inflammatory myofibroblastic tumor likely represents a heterogeneous group of spindle cell proliferations with the majority associated with $A L K$ translocations, and the remaining associated with other etiologies. Modern Pathology (2007) 20, 592-603. doi:10.1038/modpathol.3800776; published online 30 March 2007
\end{abstract}

Keywords: urinary bladder; inflammatory myofibroblastic tumor; $A L K$; fluorescence in situ hybridization; spindle cell lesion

Inflammatory myofibroblastic tumor is a rare myofibroblastic spindle cell tumor occurring at a number of anatomic sites, including the urinary bladder. ${ }^{1-9}$

Correspondence: Dr RP Ketterling, MD, Department of Laboratory Medicine and Pathology, Hilton 9, Mayo Clinic, 200 1st Street SW, Rochester 55902, MN, USA.

E-mail: ketterling.rhett@mayo.edu

${ }^{*}$ Contributed equally as senior authors.

Received 21 December 2006; revised 15 February 2007; accepted 16 February 2007; published online 30 March 2007
The vast majority of these tumors behave in a benign fashion, although occasionally tumors can recur following surgical excision. Histologically, inflammatory myofibroblastic tumor is characterized by a proliferation of spindled cells arranged in loose, variably cellular fascicles admixed with inflammatory cells including plasma cells, and lymphocytes. $^{3,6,7,10-13}$ In the urinary bladder, as in other sites, these tumors can exhibit infiltrative growth, increased cellular density, cytologic atypia, mitotic activity, mucosal ulceration and necrosis, 
mimicking malignant spindle cell tumors such as leiomyosarcoma, sarcomatoid carcinoma and rhabdomyosarcoma. ${ }^{3,6,7,10-14}$ Additionally, inflammatory myofibroblastic tumor shares immunophenotypic features with these malignant tumors, including the expression of cytokeratins, smooth muscle antigens and desmin. . $^{3,10,11,13,15-17}$ The morphologic and immunophenotypic similarities between inflammatory myofibroblastic tumor and malignant urinary bladder tumors can lead to a misdiagnosis of inflammatory myofibroblastic tumor as a highly malignant tumor, resulting in unnecessary radical surgery. Therefore, there is need for a more specific marker of inflammatory myofibroblastic tumor.

Recently, studies have shown that a subset of inflammatory myofibroblastic tumor express anaplastic lymphoma kinase (ALK-1) protein, and associated molecular cytogenetic analyses, using fluorescence in situ hybridization (FISH), has demonstrated gene rearrangements involving the $A L K$ gene locus within the spindle cell population of inflammatory myofibroblastic tumor. ${ }^{2,4,9,15,18,19}$ $A L K$, located on chromosome $2 \mathrm{p} 23$, encodes the ALK protein, a tyrosine kinase receptor, originally shown to be overexpressed in anaplastic large cell lymphoma. Rearrangements of $A L K$ with various gene partners have been identified in inflammatory myofibroblastic tumor from a number of anatomic sites. ${ }^{20-24}$ Each rearrangement is believed to result in the creation of novel gene fusions and subsequently leads to aberrant $A L K$ activation. In the urinary bladder, a few series and case studies have reported cases of inflammatory myofibroblastic tumor exhibiting $A L K$ rearrangements. ${ }^{7,10,13,19,25}$ In addition, studies have identified a subset of inflammatory myofibroblastic tumors in locations outside of the urinary bladder, demonstrating the immunophenotype of a follicular dendritic cell tumor, harboring Epstein-Barr virus (EBV) or human herpes virus-8. . $^{1,6,8,26,27}$

The objective of the present study was to determine if ALK-1 protein expression detected by immunoperoxidase methods and $A L K$ rearrangements detected by FISH are useful in discriminating between inflammatory myofibroblastic tumor and malignant spindle cell tumors of the urinary bladder. In addition, extensive immunophenotypic studies examining the expression patterns of epithelial, neural, myogenic and follicular dendritic cell markers, as well as evaluation for the presence of EBV viral sequences, were undertaken on this series of bladder neoplasms to determine their diagnostic utility.

\section{Materials and methods}

\section{Pathologic Material}

Formalin-fixed, paraffin-embedded tissue of primary urinary bladder spindle cell lesions obtained by transurethral resection or cystectomy, including
21 cases of inflammatory myofibroblastic tumor, 16 cases of leiomyosarcoma, eight cases of sarcomatoid carcinoma, three cases of embryonal rhabdomyosarcoma, one malignant solitary fibrous tumor, and four benign neoplasms (three reactive myofibroblastic proliferations and one schwannoma) were obtained from Mayo Clinic and consultation files. H\&Estained sections from each case were reviewed by a urologic pathologist (JCC).

\section{Immunohistochemistry}

Immunohistochemical evaluation was performed on $4-\mu \mathrm{m}$ formalin-fixed, paraffin-embedded tissue sections mounted on charged glass slides using the antibodies listed in Table 1.

Antigen retrieval consisted of graded alcohol deparaffinization, methanol/hydrogen peroxide block, followed by pressure treatment in buffered EDTA (pH 8) at $100^{\circ} \mathrm{C}$ for $30 \mathrm{~min}$.

Immunohistochemical staining was performed with a modified avidin-biotin-peroxidase complex method at the indicated dilutions (Table 1) using the Dako Autostainer (Dako, Carpintera, CA, USA) according to specifications. Results of immunostaining were interpreted as positive if the spindle cells exhibited specific staining for the particular antibody. Appropriate positive and negative controls were performed for each immunohistochemical stain analyzed.

\section{EBV In Situ Hybridization}

EBV RNA in situ hybridization was performed on paraffin-embedded sections from 21 inflammatory myofibroblastic tumors and 16 other spindle cell neoplasms. Oligonucleotides complementary to a portion of the EBV early RNA (EBER1 and EBER2) were applied. Cases were considered to be positive if nuclear staining was identified.

Table 1 Immunohistochemical antibodies

\begin{tabular}{lrll}
\hline Antibody & Dilution & Source & Clone \\
\hline Actin, muscle-specific & $1 / 100$ & Dako & HHF35 \\
Actin, smooth muscle & $1 / 250$ & Dako & 1A4 \\
AE1 keratin & $1 / 50$ & Zymed & AE1/AE3 \\
ALK-1 & $1 / 100$ & Dako & ALK1 \\
Caldesmon & $1 / 500$ & Dako & H-CD \\
Cam5.2 & $1 / 50$ & Becton Dickinson & Cam 5.2 \\
CD21 & $1 / 5$ & Dako & 1 F8 \\
CD34 & $1 / 100$ & Becton Dickinson & MY10 \\
CD35 & $1 / 20$ & Dako & Ber-H2 \\
C-kit & $1 / 1000$ & Dako & polyclonal \\
Clusterin & $1 / 200$ & Upstate Biotech & $41 D$ \\
Desmin & $1 / 200$ & Dako & DER11 \\
Keratin, wide spectrum & $1 / 1600$ & Dako & Polyclonal \\
Myogenin & $1 / 500$ & Dako & F5D \\
S100-protein & $1 / 1600$ & Dako & Polyclonal \\
Vimentin & $1 / 500$ & Dako & V9 \\
& & &
\end{tabular}




\section{FISH}

A dual-color, break-apart FISH probe strategy was utilized to determine $A L K$ rearrangement (Vysis Inc., Des Plaines, IL, USA). The $A L K$ region is flanked by two probes: a $250-k b$ probe labeled in Spectrum Orange (herein referred to as Red), that is telomeric to the breakpoint, and a $300-\mathrm{kb}$ probe labeled in Spectrum Green that is centromeric to the breakpoint. The close proximity of these probes results in a normal overlap of the Red (R) and Green (G) signals, referred to as a fusion (F) signal. Thus, a normal nucleus with two normal copies of the $A L K$ gene will contain two fusion signals (2F). If a genetic rearrangement has split one copy of the $A L K$ gene, the $R$ and $G$ will separate, resulting in a 1R1G1F pattern (the residual $1 \mathrm{~F}$ corresponding to the normal $A L K$ gene). Gains or losses of an intact gene region will result in gains or losses of a fusion signal (1F or $3 F$ ). Standard interphase FISH scoring criteria were applied, which dictates at least one signal width must be present between two signals to count them as two separate signals. This minimizes the impact of artifactual signal splitting.

Multiple 5- $\mu \mathrm{m}$ sections were prepared for FISH analysis studies from 78 paraffin-embedded specimens. These included 53 selected bladder tumors, comprised of 21 cases of inflammatory myofibroblastic tumor, 16 cases of leiomyosarcoma, eight cases of sarcomatoid carcinoma, three cases of embryonal rhabdomyosarcoma, one malignant solitary fibrous tumor and four benign neoplasms (three reactive myofibroblastic proliferations and one schwannoma). In addition, 25 normal urinary bladder biopsy blocks were included to help establish a normal cutoff. The first section was H\&E stained and examined to ensure that it met the standards by which the block was selected. Regions representing tumor and normal tissue were then delineated by a pathologist (JCC). Importantly, FISH analyses were performed using the sections immediately following the H\&E stained slide to minimize the effects of tumor heterogeneity.

One slide per case was heat-treated in a $90^{\circ} \mathrm{C}$ oven (30 min), deparaffinized in xylene $(2 \times 15 \mathrm{~min})$, and dehydrated $(2 \times 100 \%$ EtOH $)$. Air-dried deparaffinized sections were microwave treated ( $3 \mathrm{~min}$ ) in $10 \mathrm{mM}$ citric acid (pH 6.0), digested with $0.005 \%$ pepsin solution (Sigma-Aldrich Corp, St Louis, MO, USA) (pepsin in $0.9 \% \mathrm{NaCl}(\mathrm{pH} 1.5)$ ) (30 min) at $37^{\circ} \mathrm{C}$, sequentially dehydrated in alcohols $(70,90$ and $100 \%$ ), and again air dried. BAP $A L K$ probe working solution (Vysis Inc.) was applied to the entire tissue, and they were co-denatured within a HYBrite $^{\mathrm{TM}}$ at $80^{\circ} \mathrm{C}(5 \mathrm{~min})$. The slides were then hybridized overnight in a humidified chamber at $37^{\circ} \mathrm{C}$. Following hybridization, the slides were washed in $0.1 \% \mathrm{NP} 40 / 2 \mathrm{X}$ SSC at $72^{\circ} \mathrm{C}(2 \mathrm{~min})$, and then rinsed at room temperature in $0.1 \% \mathrm{NP} 40 / 2 \mathrm{X}$ SSC ( $1 \mathrm{~min})$. Slides were DAPI counterstained: 4',6-diamidino-2-phenyl indole dihydrochloride
(1000 ng/mL in anti-fade mounting solution) (Vysis Inc.) and then cover slipped.

All 78 specimens analyzed by FISH were processed in a blinded fashion without any link to tissue diagnosis and immunostain results until study completion. Two technologists independently analyzed each FISH slide, scanning the entire tissue section and scoring 100 representative spindleshaped nuclei (200 total). The results of each technologist were not combined until study completion to minimize any bias in scoring. Once unblinded, a normal cutoff value of $4 \%$ for the signal pattern of $1 \mathrm{R} 1 \mathrm{G} 1 \mathrm{~F}$ was calculated from the 25 normal bladder specimen results, which should reflect the frequency of artifactual splitting of the $A L K$ probes in normal bladder muscularis propria. Tumor samples were then interpreted as $A L K$ rearrangement positive if greater than $4 \%$ of the 200 cells analyzed demonstrated a separation of the $A L K$ FISH probe. Ten of the 78 samples were re-analyzed/re-probed due to poor hybridization signals and/or poor tissue morphology.

\section{Statistical Analysis}

To test if $A L K$ gene rearrangements in inflammatory myofibroblastic tumor occur more commonly in females than in males, a Fisher's exact test for count data was performed, generating a $P$-value of 0.0032 . To test for any difference in age of occurrence between patients with $A L K$-positive inflammatory myofibroblastic tumor vs $A L K$-negative inflammatory myofibroblastic tumor, a two-sample $t$-test with unequal variances yields a $P$-value of 0.167 .

\section{Results}

\section{Immunohistochemistry}

The results of immunohistochemical studies are summarized in Table 2. Cytoplasmic staining for ALK-1 was present in 13 of 21 (62\%) urinary bladder inflammatory myofibroblastic tumors (Figure 1). ALK-1 staining was not identified in any other tumor types. ALK-1 staining in inflammatory myofibroblastic tumors varied from diffuse to very focal, ranging from 5 to $75 \%$ of the tumor; median of $30 \%$ (Table 3 ). Often, only a few spindle cells demonstrated ALK-1 positivity, whereas the majority of adjacent cells were negative for ALK-1 staining. The ALK-1-positive cells typically demonstrated a slightly more atypical morphology with more abundant cytoplasm, irregular cytoplasmic contour, and large nuclei with occasional nucleoli compared to ALK-1 negative cells. Due to the focal nature of ALK-1 staining, a tumor was considered positive when any specific cytoplasmic ALK-1 staining was identified, whereas stain in intercellular areas was interpreted as negative. 
Table 2 Results of immunohistochemistry for all primary urinary bladder spindle cell lesions

\begin{tabular}{|c|c|c|c|c|c|c|c|}
\hline & Leiomyosarcoma & $\begin{array}{c}\text { Sarcomatoid } \\
\text { urothelial carcinoma }\end{array}$ & $\begin{array}{c}\text { Embryonal } \\
\text { rhabdomyosarcoma }\end{array}$ & $I M T$ & $R M P$ & $\begin{array}{l}\text { Malignant } \\
\quad \text { SFT }\end{array}$ & Schwannoma \\
\hline ALK-1 & 0/16 & $0 / 8$ & $0 / 3$ & $13 / 21$ & $0 / 3$ & $0 / 1$ & $0 / 1$ \\
\hline MSA & $15 / 16$ & $0 / 8$ & $3 / 3$ & $13 / 21$ & $0 / 3$ & $0 / 1$ & $0 / 1$ \\
\hline SMA & $14 / 16$ & $0 / 8$ & $0 / 3$ & $10 / 21$ & $1 / 3$ & $0 / 1$ & $0 / 1$ \\
\hline Desmin & $6 / 16$ & $0 / 8$ & $3 / 3$ & $1 / 21$ & $1 / 3$ & $0 / 1$ & $0 / 1$ \\
\hline Myogenin & $0 / 16$ & $0 / 8$ & $3 / 3$ & $0 / 21$ & $0 / 3$ & $0 / 1$ & $0 / 1$ \\
\hline Caldesmon & $6 / 16$ & $0 / 8$ & $0 / 3$ & $0 / 21$ & $0 / 3$ & $0 / 1$ & $0 / 1$ \\
\hline Vimentin & $15 / 16$ & $7 / 8$ & $2 / 3$ & $20 / 21$ & $3 / 3$ & $0 / 1$ & $1 / 1$ \\
\hline c-kit & $0 / 16$ & $0 / 8$ & $1 / 3$ & $0 / 21$ & $0 / 3$ & $0 / 1$ & $0 / 1$ \\
\hline CAM5.2 & $2 / 16$ & $3 / 8$ & $0 / 3$ & $8 / 21$ & $0 / 3$ & $0 / 1$ & $0 / 1$ \\
\hline AE1/AE3 & $1 / 16$ & $8 / 8$ & $0 / 3$ & $13 / 21$ & $0 / 3$ & $0 / 1$ & $0 / 1$ \\
\hline WS Keratin & $0 / 16$ & $4 / 8$ & $0 / 3$ & $2 / 21$ & $0 / 3$ & $0 / 1$ & $0 / 1$ \\
\hline CD34 & $0 / 16$ & $0 / 8$ & $0 / 3$ & $0 / 21$ & $0 / 3$ & $1 / 1$ & $0 / 1$ \\
\hline CD21 & $0 / 16$ & $0 / 8$ & $0 / 3$ & $0 / 21$ & $0 / 3$ & $0 / 1$ & $0 / 1$ \\
\hline Clusterin & $3 / 16$ & $1 / 8$ & $0 / 3$ & $5 / 21$ & $1 / 3$ & $0 / 1$ & $1 / 1$ \\
\hline CD35 & $0 / 16$ & $0 / 8$ & $0 / 3$ & $0 / 21$ & $0 / 3$ & $0 / 1$ & $0 / 1$ \\
\hline
\end{tabular}

MSA, muscle specific actin; SMA, smooth muscle actin; WS keratin, wide spectrum keratin; RMP, reactive myofibroblastic proliferation; SFT, solitary fibrous tumor.

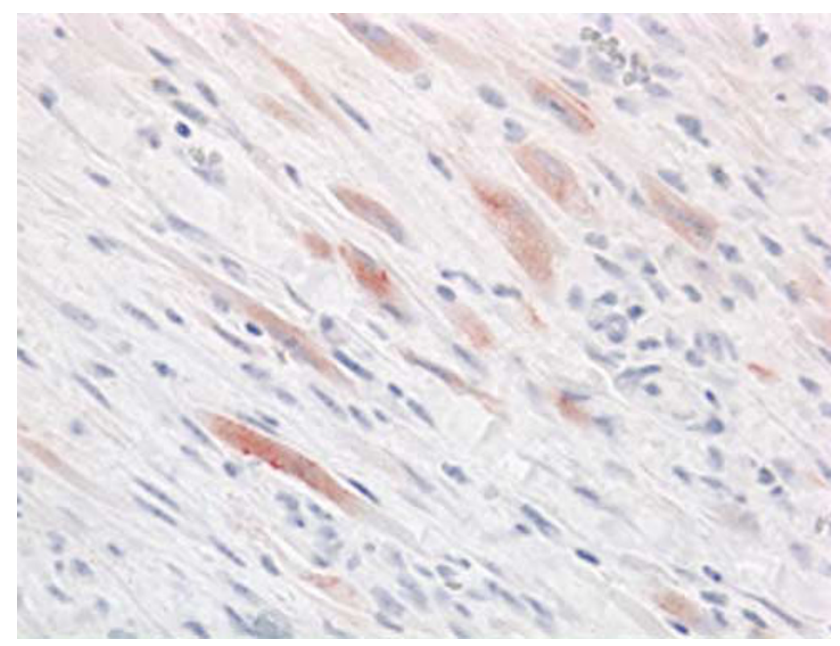

Figure 1 Immunohistochemical analysis for ALK-1 expression in inflammatory myofibroblastic tumor of urinary bladder. ALK-1 immunostaining in inflammatory myofibroblastic tumor of urinary bladder shows a cytoplasmic staining pattern specific to a population of spindle cells within the lesion.

Staining for muscle markers (muscle-specific actin (MSA), smooth muscle actin (SMA), desmin, myogenin and caldesmon) were uniformly identified in leiomyosarcoma and rhabdomyosarcoma. Approximately $50 \%$ of inflammatory myofibroblastic tumors exhibited staining for MSA and SMA, whereas only a single tumor was positive for desmin (Table 2). All inflammatory myofibroblastic tumors were negative for myogenin and caldesmon. Staining for epithelial markers (CAM 5.2, keratin AE1/ AE3 and wide spectrum keratin) also showed a variable pattern of expression in various tumors, but was detected consistently in sarcomatoid carcinoma. Cytokeratin expression was also identified in inflammatory myofibroblastic tumor with over $60 \%$ of all tumors staining for AE1/AE3. Eleven of 21 inflammatory myofibroblastic tumors were positive for both muscle antigens (SMA or MSA) and cytokeratins (CAM 5.2, AE1/AE3 or wide spectrum keratin). However, five of 21 cases of inflammatory myofibroblastic tumor $(24 \%)$ stained positively for cytokeratins without muscle antigen expression and four of 21 (19\%) stained positively for muscle antigens without positive staining for cytokeratins. Staining for follicular dendritic cell markers, CD 21 and CD 35, was uniformly negative in all lesions. Positive staining for the follicular dendritic cell marker, clusterin, typically showed a cytoplasmic staining pattern associated with scattered foci of spindled cells and was identified to a variable extent in all tumors with the exception of rhabdomyosarcoma. However, no tumor demonstrated strong or diffuse staining. Immunostaining demonstrated no correlation between staining pattern for ALK-1 and any other marker in inflammatory myofibroblastic tumor.

\section{EBV In Situ Hybridization}

All tumors in the study were negative for EBV RNA by in situ hybridization.

\section{FISH}

Once the results were unblinded, it was determined that no separation of the $A L K$ FISH signal was observed in any of the 25 normal bladder biopsy specimens. Of the 21 cases of bladder inflammatory myofibroblastic tumor, 14 demonstrated rearrangement of $A L K$ and seven yielded normal results (Table 3). The seven $A L K$-negative inflammatory myofibroblastic tumors demonstrated intact fusion signals in all nuclei (Figure 2a). Separation of $A L K$ was limited to the spindle cells within each tumor specimen and varied widely in the percentage of 
Table 3 Clinicopathologic findings for IMT cases

\begin{tabular}{|c|c|c|c|c|c|c|}
\hline Case & Age & Gender & $\begin{array}{l}\text { ALK-1 expression } \\
\text { (\% of tumor if positive) }\end{array}$ & $\begin{array}{l}\text { FISH for ALK translocation } \\
\text { (\% of tumor if positive) }\end{array}$ & Treatment & $\begin{array}{l}\text { Follow-up (months); } \\
\text { patient status }\end{array}$ \\
\hline 1 & 36 & $\mathrm{M}$ & Negative & Negative & Partial cystectomy & No F/U available \\
\hline 2 & 40 & $\mathrm{M}$ & Negative & Negative & TUR & No F/U available \\
\hline 3 & 41 & M & Negative & Negative & TUR & No F/U available \\
\hline 4 & 56 & $\mathrm{M}$ & Negative & Negative & TUR & 7-no recurrence \\
\hline 5 & 66 & $\mathrm{M}$ & Negative & Negative & TUR & 42-no recurrence \\
\hline 6 & 83 & $\mathrm{~F}$ & Negative & Negative & TUR & 7-no recurrence \\
\hline 7 & 88 & $\mathrm{M}$ & Negative & Negative & TUR & No F/U available \\
\hline 8 & 26 & $\mathrm{M}$ & Positive (40) & Positive (72.5) & TUR & No F/U available \\
\hline 9 & 29 & $\mathrm{~F}$ & Positive (5) & Positive (49.5) & TUR & 18-no recurrence \\
\hline 10 & 29 & $\mathrm{~F}$ & Positive (5) & Positive (57) & TUR & No F/U available \\
\hline 11 & 32 & $\mathrm{~F}$ & Positive (75) & Positive (69) & TUR & $64-$ no recurrence \\
\hline 12 & 38 & $\mathrm{~F}$ & Positive (10) & Positive (28) & TUR & 34-no recurrence \\
\hline 13 & 39 & $\mathrm{~F}$ & Positive (50) & Positive $(4)^{\mathrm{a}}$ & TUR & No F/U available \\
\hline 14 & 40 & $\mathrm{~F}$ & Positive (30) & Positive (37.5) & TUR & 59-no recurrence \\
\hline 15 & 42 & $\mathrm{M}$ & Positive (40) & Positive (55) & TUR & No F/U available \\
\hline 16 & 44 & $\mathrm{~F}$ & Positive (5) & Positive (42.5) & Partial cystectomy & No F/U available \\
\hline 17 & 47 & $\mathrm{~F}$ & Positive (20) & Positive (40.5) & TUR & 70-no recurrence \\
\hline 18 & 51 & $\mathrm{~F}$ & Positive (50) & Positive (26) & TUR & 13-no recurrence \\
\hline 19 & 67 & $\mathrm{~F}$ & Positive (20) & Positive (12.5) & TUR & No F/U available \\
\hline 20 & 69 & $\mathrm{~F}$ & Positive (30) & Positive (53) & TUR & 13-no recurrence \\
\hline 21 & 77 & $\mathrm{~F}$ & Negative & Positive (21) & TUR & 70 -no recurrence \\
\hline
\end{tabular}

TUR, transurethral resection.

${ }^{\mathrm{a}}$ Large abnormal spindle cells degraded.

spindle cells involved (12.5-72.5\%). The split signals were widely separated within the nuclei in 13 of the tumors, suggesting a possible translocation mechanism for these rearrangements (Figure 2b). Rare nuclei in the $A L K$-positive inflammatory myofibroblastic tumor demonstrated gains or losses of the split $R$ and $G$ or the intact $F$ signals (Figure 2e and f). In a single case (inflammatory myofibroblastic tumor case 18), a very small split signal was observed (Figure 2c), such that the R and G signals were separated, but the signals tracked together in the nucleus. This unusual result suggests a possible insertion or inversion event in $A L K$ in this tumor. Four of the inflammatory myofibroblastic tumor had a normal $2 \mathrm{~F}$ result in the spindle cells and four inflammatory myofibroblastic tumor had $3 \mathrm{~F}-4 \mathrm{~F}$ in the spindle cells (Figure 2a and d).

Of the 32 non-inflammatory myofibroblastic tumor specimens included in the study, no samples displayed a split-signal pattern. Of the 16 leiomyosarcomas, 12 demonstrated gains of intact fusion signals (3F-6F) (Figure 3b), three demonstrated loss of one fusion signal (1F) (Figure 3c) in greater than $90 \%$ of spindle-shaped nuclei and one had a normal $2 \mathrm{~F}$ result (Figure 3a). All three embryonal rhabdomyosarcomas and the one malignant solitary fibrous tumor demonstrated $3 \mathrm{~F}-4 \mathrm{~F}$ in the spindle cells. The spindle cells in the schwannoma and the three reactive myofibroblastic proliferations demonstrated a normal result of $2 \mathrm{~F}$ signals. The most unusual result was observed in the case of a sarcomatoid carcinoma, which demonstrated amplification of an intact fusion, with 7-25 fusion signals per spindleshaped nucleus (Figure 3d). The remaining seven sarcomatoid carcinomas demonstrated either nor- mal results of $2 \mathrm{~F}$ (in four cases), or $3 \mathrm{~F}$ to $4 \mathrm{~F}$ (in three cases).

\section{Correlation between Immunohistochemistry and FISH}

Once unblinded and compared to ALK-1 immunohistochemistry results, it was determined that $A L K$ FISH and ALK-1 immunostain results were concordant for 20 of the 21 inflammatory myofibroblastic tumor specimens (95\%). These results included seven inflammatory myofibroblastic tumors for which both FISH and immunostain results were negative and 13 inflammatory myofibroblastic tumor cases that yielded positive results for both FISH and immunostain. A single discordant case (inflammatory myofibroblastic tumor case 21) showed positive FISH results and was negative for ALK-1 immunostain. Based on the positive FISH result, the ALK-1 immunostain was repeated and remained negative. Examination of H\&E sections (Figure 4) revealed inflammatory myofibroblastic tumors with and without $A L K$ rearrangements to be histologically indistinguishable.

The percentage of ALK-positive spindle cells, as detected by immunostain vs FISH, was quite variable in each tumor and showed very little correlation (Table 3). The three IMT tumors with the lowest ALK-positive immunostain percentages (cases 9, 10 and 16), each had high levels of ALK-positive FISH results. Similarly, the tumors with the larger percentages of ALK-positive cells by immunostain often demonstrated a low percentage of ALKpositive cells by FISH (cases 13 and 18).

With regard to all other immunohistochemical stains, no difference in staining pattern was noted 

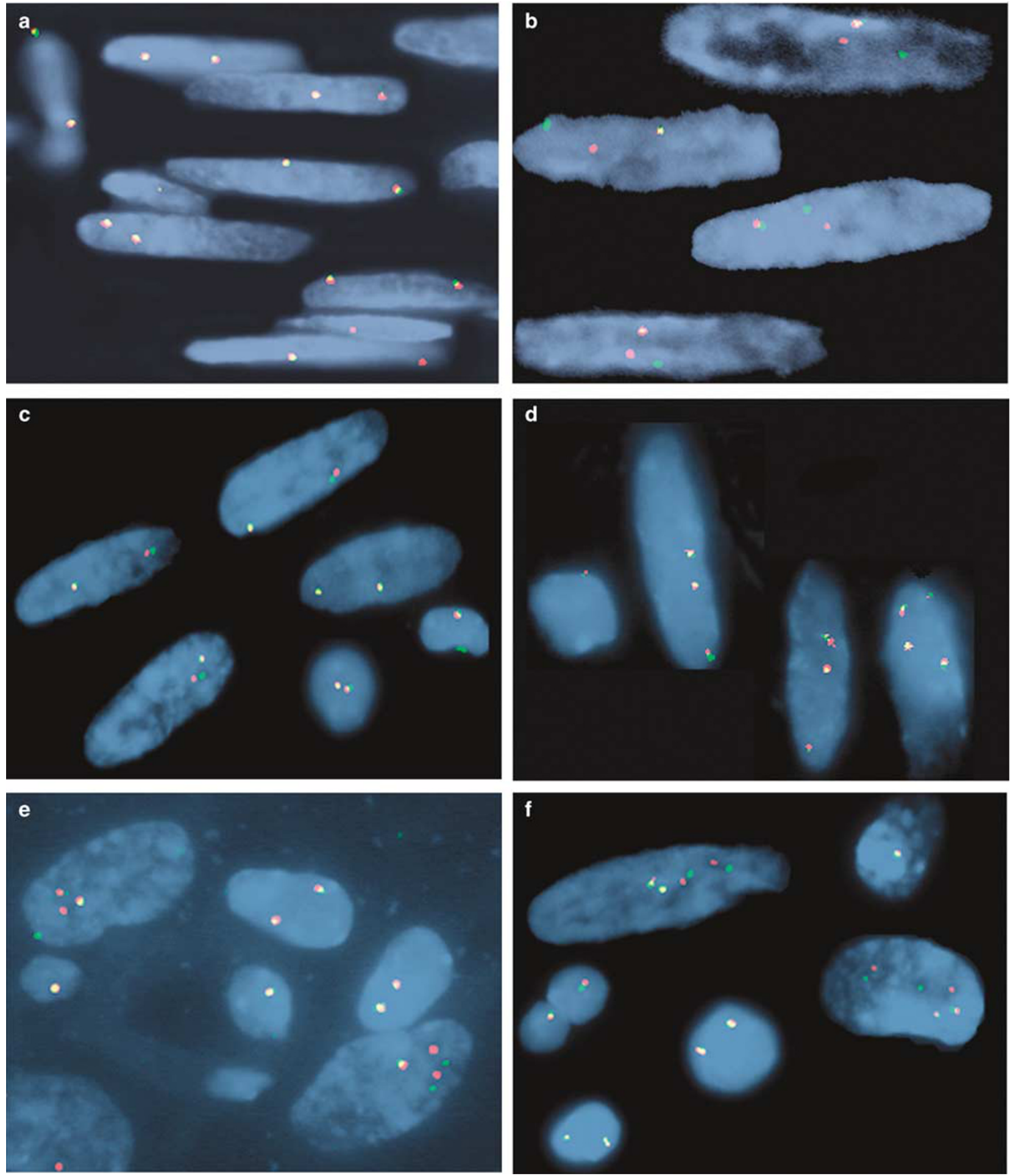

Figure 2 FISH analysis of bladder inflammatory myofibroblastic tumor using a break-apart strategy with probes flanking both telomeric and centromeric sides of the $A L K$ locus. (a) Normal signal pattern 2F; (b) abnormal with typical split-signal pattern, 1R1G1F; (c) abnormal with atypical/small split-signal pattern, 1R1G1F; (d) extra intact signals, 3F-4F; abnormal atypical patterns (e) 2R2G1F and (f) 2R2G2F. Bold results indicate immunohistochemical stains which may have diagnostic utility in inflammatory myofibroblastic tumor.

between inflammatory myofibroblastic tumor with and without $A L K$ rearrangements. Also, no correlation was identified between $A L K$ rearrangements and coexpression of muscle antigens and cytokeratins; eight cases of inflammatory myofibroblastic tumor positive for $A L K$ rearrangement expressed both muscle antigens and cytokeratins and six cases of inflammatory myofibroblastic tumor without $A L K$ rearrangements simultaneously expressed muscle markers and cytokeratins. 

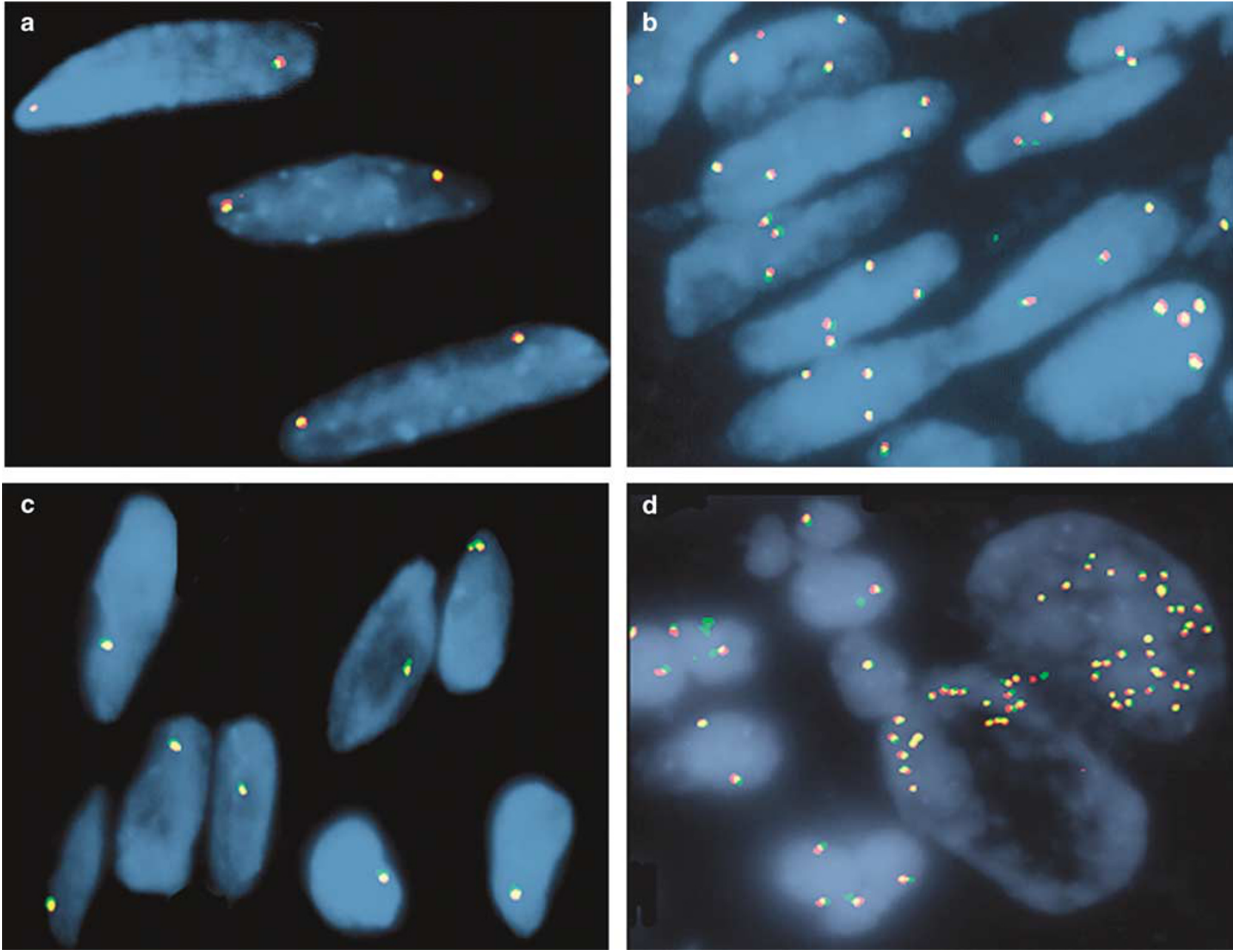

Figure 3 Representative images from non-inflammatory myofibroblastic tumor spindle cell tumor FISH results. (a) Normal signal pattern $2 \mathrm{~F}$; (b) extra intact signals, 3F-4F; (c) loss of fusion, 1F; and (d) amplification of intact fusion signals.

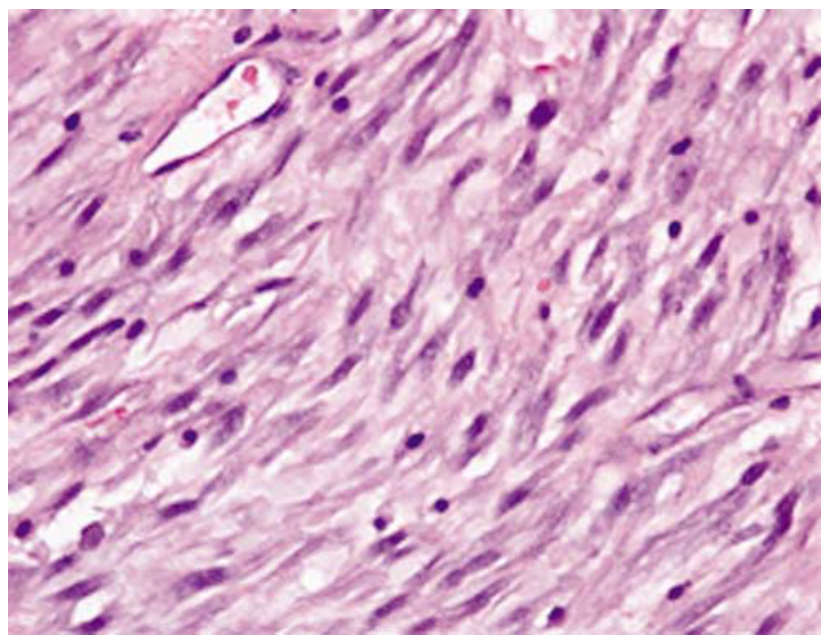

Figure 4 H\&E section of inflammatory myofibroblastic tumor with $A L K$ rearrangement.

Clinical features and association with immunohistochemistry/FISH results

Patients with inflammatory myofibroblastic tumor of the urinary bladder ranged from 26 to 88 years with a median age of 41 years (Table 3). The inflammatory myofibroblastic tumors were slightly more common in females than males (1.6:1.0). Clinical follow-up was obtained for 11 patients with inflammatory myofibroblastic tumor with a mean post-surgical follow-up of 36 months (range 7-70 months; median 34 months). All tumors behaved in a benign fashion and no tumors recurred or metastasized.

There were no age differences between patients whose tumors did and did not exhibit $A L K$ rearrangements $(P=0.167)$. However, $A L K$ rearrangements were significantly more common in inflammatory myofibroblastic tumors occurring in women vs men with a ratio of $6: 1(P=0.0032)$.

\section{Discussion}

Inflammatory myofibroblastic tumor is an unusual benign neoplasm of the urinary bladder, characterized by a spindle cell proliferation accompanied by a mixed inflammatory infiltrate comprised primarily of lymphocytes and plasma cells. Inflammatory 
myofibroblastic tumor exhibits morphologic and immunophenotypic overlap with malignant spindle cell tumors of the urinary bladder and diagnostic distinction from these tumors can be problematic. . $^{3,6,70-14,16,17}$ Both epithelial and myogenic markers can be expressed in inflammatory myofibroblastic tumor and may lead to a misdiagnosis of sarcomatoid carcinoma, leiomyosarcoma or rhabdomyosarcoma. ${ }^{6,7,11,13}$ This study confirms the utility of the detection of ALK-1 protein by immunoperoxidase methods and $A L K$ rearrangements by FISH and the limited value of immunohistochemical markers for epithelial, myogenic and follicular dendritic differentiation in the separation of inflammatory myofibroblastic tumors from malignant spindle cell tumors of the urinary bladder.

Originally identified as a protein overexpressed in anaplastic large-cell lymphoma, ALK-1 has subsequently been shown to be overexpressed in a substantial proportion of inflammatory myofibroblastic tumors of various anatomic locations, ${ }^{1-9,15}$ including the urinary bladder. ${ }^{3,7,10-14,16,19,25}$ Several studies have demonstrated a variable degree of cytoplasmic immunohistochemical staining for ALK-1. In inflammatory myofibroblastic tumor of the urinary bladder, positivity for ALK-1 by immunohistochemistry ranges from 33 to $89 \%$, whereas ALK-1 protein expression in leiomyosarcoma and sarcomatoid urothelial carcinoma has not been reported, suggesting that ALK-1 immunohistochemical studies may be useful in the differentiation of inflammatory myofibroblastic tumor from other spindle cell lesions in the urinary bladder. Similar to previous reports, in the present study, we noted ALK-1 expression in $62 \%$ (13 of 21 cases) of bladder inflammatory myofibroblastic tumor cases. ${ }^{7,13-15,19}$ ALK-1 positivity by immunohistochemistry was not detected in leiomyosarcoma or sarcomatoid carcinoma. Previous studies have also reported ALK expression in both alveolar and embryonal rhabdomyosarcoma, using a polyclonal ALK antibody. ${ }^{28}$ However, the three cases of embryonal rhabdomyosarcoma in this series were negative.

The presence of clonal rearrangements involving the $A L K$ gene has been identified in a subgroup of inflammatory myofibroblastic tumors. ${ }^{4,5,7,9,20-25,29-32}$ Cytogenetic and molecular analyses have demonstrated a number of $A L K$ fusion gene partners, including RANBP2-ALK, TPM3-ALK, TPM4-ALK, CLTCALK, CARS-ALK and SEC31L1-ALK. ${ }^{5,9,21,25,29,31}$ In the present study, $A L K$ rearrangements were identified in 14 of 21 cases of inflammatory myofibroblastic tumor of the urinary bladder. These findings are similar to recent studies that identified $A L K$ rearrangements by FISH in a few series of urinary bladder inflammatory myofibroblastic tumors. ${ }^{7,10,19,23,25}$ Once unblinded and correlated with the ALK-1 immunohistochemical staining, all inflammatory myofibroblastic tumor cases in our study that demonstrated ALK-1 expression also exhibited $A L K$ rearrangements, although the percen- tage of positive spindle cells varied significantly between the two methods (Table 3). These findings suggest that the presence of $A L K$ expression in urinary bladder inflammatory myofibroblastic tumor is primarily, and perhaps solely due to a translocation or inversion process resulting in the aberrant expression of $A L K$ and conversely these data suggest smaller molecular activating point mutations, deletions or insertions must be rare in these tumors.

Thirteen of the 14 cases with positive FISH results had typical split signals identified within the neoplastic spindle-shaped nuclei, such that the split signals were widely separated within the neoplastic cells (Figure 2b). The sole exception (inflammatory myofibroblastic tumor case 18) demonstrated an atypical, but reproducible 'small-split' signal, which was present in the majority of spindled nuclei (Figure 2c). This signal pattern could easily be missed without careful analysis of the spindle cell nuclei, as artifactual splitting of the $A L K$ FISH signal is occasionally seen in some nuclei. We felt very confident with this result, however, since a high percentage of nuclei $(26 \%)$ had this atypical split result, it was detected independently by both FISH technologists scoring the sample and once unblinded, matched the positive ALK-1 immunostaining results. As these split signals are consistently tracking together in the interphase nuclei, this particular signal pattern strongly suggests an insertion or inversion mechanism for $A L K$ gene activation in this tumor. Activation of ALK via rearrangement with other genes on chromosome 2 has been described previously and may account for this observation. ${ }^{33}$

A few inflammatory myofibroblastic tumor cases had occasional spindle cells with atypical splitsignal patterns in addition to the typical $1 \mathrm{R} 1 \mathrm{G} 1 \mathrm{~F}$. These various signal patterns correspond to gains and/or losses of the $A L K$ gene. Some of the abnormal patterns indicated a duplication of the split signals that may represent a tetraploid component for these tumors (Figure 2e and f).

The extent of the immunohistochemical detection of ALK-1 in positive cases varied widely case to case with ALK-1 staining, ranging from isolated clusters of spindle cells to the majority of cells $(5-75 \%)$. A similarly variable distribution was reflected in FISH analysis for $A L K$ rearrangements $(4-72.5 \%)$. The significance of this variability is unknown, but it is critical to appreciate this variability in the assessment of both the immunostain and FISH assay. In contrast to the majority of FISH assays that consist of simply scoring consecutive nuclei with fluorescent signals, this particular FISH assay required significant technologist effort and diligence in identifying the presence of positive nuclei. First, only spindleshaped cells were scored for the presence of intact fusions and/or split signals. As standard 5- $\mu \mathrm{m}$ tissue sections were used, cut artifact was often encountered in these larger spindle-shaped cells, which 
artificially resulted in loss of intact fusion signals. This artifact is a common occurrence in paraffinbased tissue FISH applications, but was particularly problematic in this assay as only the larger spindle shaped cells were assessed. Some of the tumors had an abundance of spindle-shaped cells for which only a small proportion demonstrated a positive split-signal result, whereas others had an abundance of positive nuclei (range of 12.5 to $72.5 \%$ positive) (Figure 5). Thus, a quick cursory scan of a large tissue section could easily result in a false-negative FISH result. Each case required a very careful examination of the entire tissue to detect the presence of a sparse population of spindle cells exhibiting $A L K$ rearrangements. It is of note that one inflammatory myofibroblastic tumor case (case 21) repeatedly failed to show ALK-1 expression by immunostaining, yet harbored a typical split FISH signal in $21 \%$ of spindle cells, indicating an $A L K$ rearrangement. All other cases of inflammatory myofibroblastic tumor that were negative for ALK-1 immunohistochemical expression also failed to show $A L K$ rearrangements by FISH. It is unclear why in one case an $A L K$ rearrangement was not associated with apparent protein expression.

Although $A L K$ FISH did not display any split signal results in the 32 non-inflammatory myofibroblastic tumor specimens, gains or losses of intact $A L K$ fusion signals were commonly observed (Figure 3b). However, gains or losses of intact fusion signals, or normal $2 \mathrm{~F}$ results, cannot be used to rule-in or rule-out inflammatory myofibroblastic tumor or any other spindle tumors of the bladder as these FISH patterns were observed in both inflammatory myofibroblastic tumors lacking $A L K$ separation and other malignant spindle tumors. Of note, one case of sarcomatoid carcinoma demonstrated an apparently amplified, but intact, $A L K$ FISH signal (Figure 3d). Amplification of a gene region is often associated with overexpression of the associated protein, although this particular tumor was not positive for ALK-1 expression by immunohistochemistry. As no split $A L K$ signals were identified in any of the 32 non-inflammatory myofibroblastic tumors, this suggests a positive $A L K$ result in a spindle cell lesion of

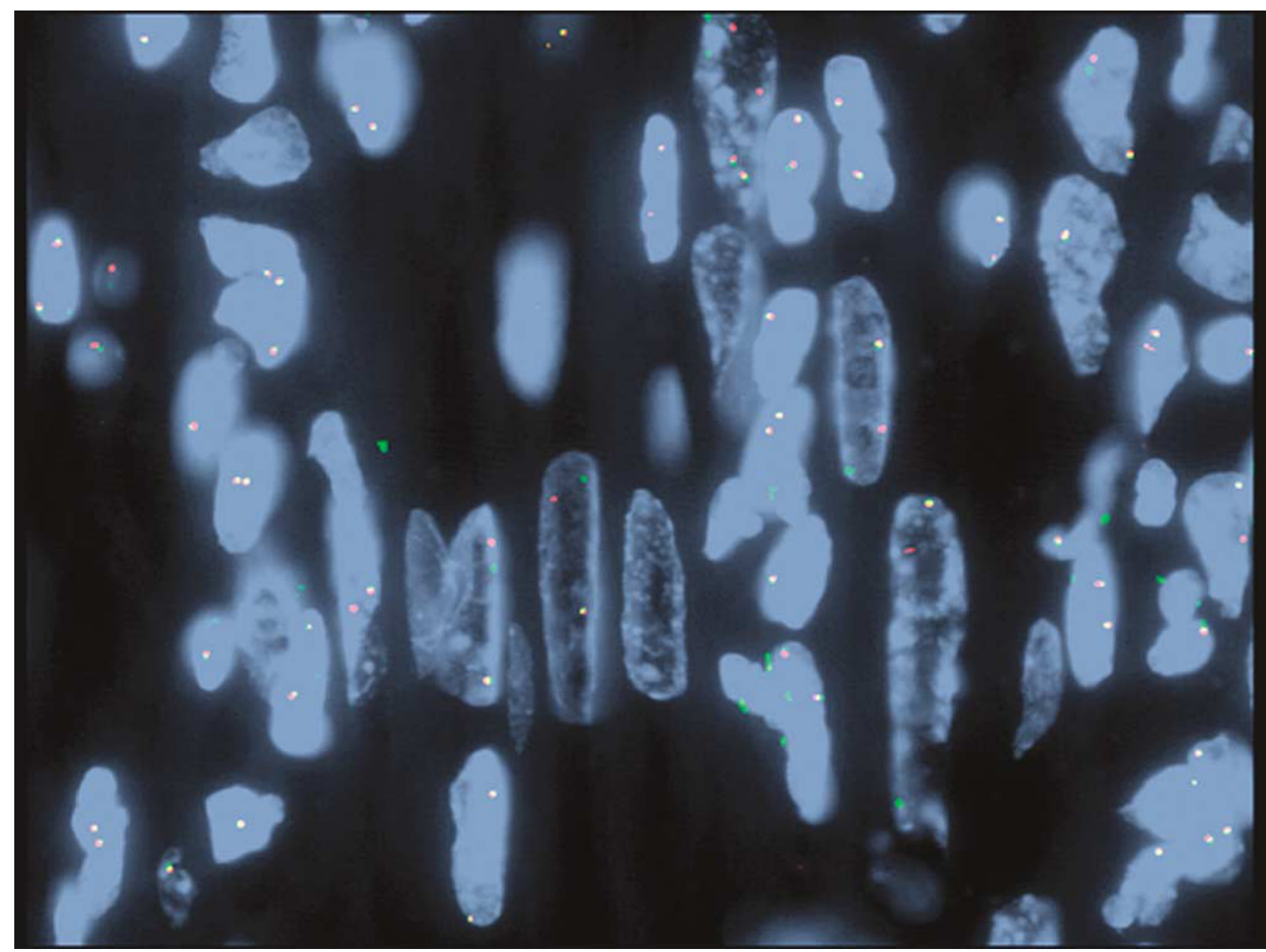

Figure 5 Field of view of inflammatory myofibroblastic tumor with typical split-signal pattern (1R1G1F) observed in the spindle cell nuclei and normal signal pattern (2F) in the other cell types. 
the urinary bladder is likely specific for inflammatory myofibroblastic tumor.

Presence or absence of $A L K$ translocations did not, in this study, appear to impact patient prognosis as eight $A L K$-rearrangement positive and three $A L K$ rearrangement negative cases had clinical follow-up and no tumors recurred following complete removal. However, inflammatory myofibroblastic tumors exhibiting $A L K$ translocations demonstrated a strong predilection for females, whereas the $A L K$-rearrangement negative inflammatory myofibroblastic tumors were predominately identified in males $(P=0.0032)$. It is interesting to postulate the gender association of $A L K$-rearrangement positive inflammatory myofibroblastic tumor in females may correspond to a unique etiology relative to these tumor growths in males.

Previous studies of inflammatory myofibroblastic tumor in various anatomic locations have suggested that lesions that carry an $A L K$ translocation and/or ALK-1 protein expression occur in patients less than 40 years of age, whereas those that do not show $A L K$ translocations or ALK-1 protein expression are typically diagnosed in older patients. ${ }^{4,17,18,25}$ In this study, ALK-1 expression or ALK rearrangements were not associated with age. Although the median age of $A L K$-rearrangement positive cases was slightly lower than the median age of $A L K$-rearrangement negative cases, the age ranges were similar $(P=0.167) \quad$ (Table 3). Both younger and older patients were represented in both groups, with one $A L K$-positive tumor occurring in a 77-year-old and one $A L K$-negative tumor diagnosed in a 36-year-old.

Previously, it has been suggested that lesions categorized together as inflammatory myofibroblastic tumor may, in fact, represent more than one distinct entity. ${ }^{18,34}$ Our findings suggest that there are at least two types of inflammatory myofibroblastic tumor in the urinary bladder; tumors that arise in association with $A L K$ rearrangements and tumors with an apparently separate etiology. A few inflammatory myofibroblastic tumor cases have been described in the literature implicating additional genetic mechanisms. ${ }^{34}$ Further studies are necessary to determine if inflammatory myofibroblastic tumor without rearrangements in $A L K$ are due to a single underlying mechanism or if these tumors arise via multiple pathways.

Despite the identification of characteristic genetic changes associated with inflammatory myofibroblastic tumors, questions regarding etiology remain to be clarified. Recent studies examining follicular dendritic cell markers have suggested that inflammatory myofibroblastic tumor may be derived from follicular dendritic cells and may be related to infection with EBV. ${ }^{1,6,8,26,35}$ In the present study, however, inflammatory myofibroblastic tumor with and without $A L K$ abnormalities did not express the follicular dendritic cell antigens, CD23 or CD35, and all cases were negative for EBV by in situ hybridization. Additionally, although clusterin positive cells were detected in some inflammatory myofibroblastic tumor, these cells were relatively sparse and not in a pattern characteristic of follicular dendritic cell tumors. ${ }^{27}$ Further, a similar frequency of and pattern for clusterin expression was identified in leiomyosarcoma and sarcomatoid carcinoma, suggesting follicular dendritic cells are not the origin of the urinary bladder inflammatory myofibroblastic tumor. Therefore, it is likely that cases of inflammatory myofibroblastic tumor in the urinary bladder represent an entity distinct from those inflammatory myofibroblastic tumors that are of follicular dendritic cell origin or are associated with EBV infection. It is clear that these tumors exhibit myofibroblastic differentiation with expression of muscle-specific antigens. In addition, these tumors can coexpress cytokeratins, and this can be one helpful feature in distinguishing inflammatory myofibroblastic tumor from sarcomatoid carcinoma and leiomyosarcoma that both typically lack coexpression of these antigens. However, such a dual expression pattern should also elicit the consideration of a prostatic leiomyosarcoma with extension into the urinary bladder or a primary sarcomatoid urothelial carcinoma with muscle differentiation, as these lesions have been shown to coexpress cytokeratins and myogenic markers as well. ${ }^{36,37}$

In summary, we expand the literature with a large series of urinary bladder inflammatory myofibroblastic tumors harboring $A L K$ gene rearrangements, and confirm the utility of immunohistochemical detection of ALK-1 protein and FISH analysis for $A L K$ rearrangements in distinguishing inflammatory myofibroblastic tumor from other malignant spindle cell tumors of the urinary bladder. We suggest both methods of detection should be concomitantly utilized as the percentage of either immunostain or FISH-positive cells is highly variable and a falsenegative result could be easily generated. It is hypothesized that at least two mechanisms are responsible for inflammatory myofibroblastic tumor of the urinary bladder with the most common mechanism associated with $A L K$ gene rearrangements. FISH confirmed that $A L K$ translocations or inversions may be the sole mechanism for activation of this gene in inflammatory myofibroblastic tumor, although very careful examination of the entire tissue section is required for identification of ALK rearrangements in spindle cells. Although an ageassociated occurrence of $A L K$-rearrangement positive inflammatory myofibroblastic tumor in the bladder was not observed, a statistically significant gender predilection for $A L K$-rearrangement positive inflammatory myofibroblastic tumor in females vs $A L K$-rearrangement negative inflammatory myofibroblastic tumor in males was identified $(P=0.0032)$. In the diagnosis of inflammatory myofibroblastic tumor, immunohistochemistry may provide additional useful information, particularly the coexpression of cytokeratins and actins. However, these stains performed in isolation have the potential to result in a misdiagnosis of sarcomatoid carcinoma or leiomyosarcoma. 


\section{References}

1 Arber DA, Kamel OW, van de Rijn M, et al. Frequent presence of the Epstein-Barr virus in inflammatory pseudotumor. Hum Pathol 1995;26:1093-1098.

2 Cessna MH, Zhou H, Sanger WG, et al. Expression of ALK1 and p80 in inflammatory myofibroblastic tumor and its mesenchymal mimics: a study of 135 cases. Mod Pathol 2002;15:931-938.

3 Coffin CM, Fletcher JA. Inflammatory myofibroblastic tumour In: Fletcher CDM, Unni KK, Mertens F (eds). World Health Organization Classification of Tumours: Tumours of Soft Tissue and Bone. IARC Press: Lyons, France, 2002;91-93.

4 Coffin CM, Patel A, Perkins S, et al. ALK1 and p80 expression and chromosomal rearrangements involving 2p23 in inflammatory myofibroblastic tumor. Mod Pathol 2001;14:569-576.

5 Lawrence B, Perez-Atayde A, Hibbard MK, et al. TPM3-ALK and TPM4-ALK oncogenes in inflammatory myofibroblastic tumors. Am J Pathol 2000;157: 377-1384.

6 Mergan F, Jaubert F, Sauvat F, et al. Inflammatory myofibroblastic tumor in children: clinical review with anaplastic lymphoma kinase, Epstein-Barr virus, and human herpesvirus 8 detection analysis. J Pediatr Surg 2005;40:1581-1586.

7 Montgomery EA, Shuster DD, Burkart AL, et al. Inflammatory myofibroblastic tumors of the urinary tract: a clinicopathologic study of 46 cases, including a malignant example of inflammatory fibrosarcoma and a subset associated with high-grade urothelial carcinoma. Am J Surg Pathol 2006;30:1502-1512.

8 Neuhauser TS, Derringer GA, Thompson LD, et al. Splenic inflammatory myofibroblastic tumor (inflammatory pseudotumor): a clinicopathologic and immunophenotypic study of 12 cases. Arch Pathol Lab Med 2001;125:379-385.

9 O’Malley DP, Poulos C, Czader M, et al. Intraocular inflammatory myofibroblastic tumor with ALK overexpression. Arch Pathol Lab Med 2004;128:e5-e7.

10 Harik LR, Merino C, Coindre JM, et al. Pseudosarcomatous myofibroblastic proliferations of the bladder: a clinicopathologic study of 42 cases. Am J Surg Pathol 2006;30:787-794.

11 Iczkowski KA, Shanks JH, Gadaleanu $\mathrm{V}$, et al. Inflammatory pseudotumor and sarcoma of urinary bladder: differential diagnosis and outcome in thirtyeight spindle cell neoplasms. Mod Pathol 2001;14: 1043-1051.

12 Lundgren L, Aldenborg F, Angervall L, et al. Pseudomalignant spindle cell proliferation of the urinary bladder. Hum Pathol 1994;25:181-191.

13 Tsuzuki T, Magi-Galluzzi C, Epstein JI. ALK-1 expression in inflammatory myofibroblastic tumor of the urinary bladder. Am J Surg Pathol 2004;28:1609-1614.

14 Hirsch MS, Dal Cin P, Fletcher CD. ALK expression in pseudosarcomatous myofibroblastic proliferations of the genitourinary tract. Histopathology 2006;48: $569-578$.

15 Cook JR, Dehner LP, Collins MH, et al. Anaplastic lymphoma kinase (ALK) expression in the inflammatory myofibroblastic tumor: a comparative immunohistochemical study. Am J Surg Pathol 2001;25:1364-1371.

16 Weidner N. Inflammatory (myofibroblastic) pseudotumor of the bladder: a review and differential diagnosis. Adv Anat Pathol 1995;1995:362-375.
17 Young RH, Wick MR, Mills SE. Sarcomatoid carcinoma of the urinary bladder: a clinicopathologic analysis of 12 cases and review of the literature. Am J Clin Pathol 1988;90:651-653.

18 Chan JK, Cheuk W, Shimizu M. Anaplastic lymphoma kinase expression in inflammatory pseudotumors. Am J Surg Pathol 2001;25:761-768.

19 Freeman A, Geddes N, Munson P, et al. Anaplastic lymphoma kinase (ALK 1) staining and molecular analysis in inflammatory myofibroblastic tumours of the bladder: a preliminary clinicopathological study of nine cases and review of the literature. Mod Pathol 2004;17:765-771.

20 Griffin CA, Hawkins AL, Dvorak C, et al. Recurrent involvement of 2p23 in inflammatory myofibroblastic tumors. Cancer Res 1999;59:2776-2780.

21 Ma Z, Hill DA, Collins MH, et al. Fusion of ALK to the Ran-binding protein 2 (RANBP2) gene in inflammatory myofibroblastic tumor. Genes Chromosomes Cancer 2003;37:98-105.

22 Panagopoulos I, Nilsson T, Domanski HA, et al. Fusion of the SEC31L1 and ALK genes in an inflammatory myofibroblastic tumor. Int J Cancer 2006;118:1181-1186.

23 Sirvent N, Hawkins AL, Moeglin D, et al. ALK probe rearrangement in a $t(2 ; 11 ; 2)(p 23 ; p 15 ; q 31)$ translocation found in a prenatal myofibroblastic fibrous lesion: toward a molecular definition of an inflammatory myofibroblastic tumor family? Genes Chromosomes Cancer 2002;31:85-90.

24 Yousem SA, Shaw H, Cieply K. Involvement of 2p23 in pulmonary inflammatory pseudotumors. Hum Pathol 2001;32:428-433.

25 Debiec-Rychter M, Marynen P, Hagemeijer A, et al. ALK-ATIC fusion in urinary bladder inflammatory myofibroblastic tumor. Genes Chromosomes Cancer 2003;38:187-190.

26 Cheuk W, Chan JK, Shek TW, et al. Inflammatory pseudotumor-like follicular dendritic cell tumor: a distinctive low-grade malignant intra-abdominal neoplasm with consistent Epstein-Barr virus association. Am J Surg Pathol 2001;25:721-731.

27 Grogg KL, Macon WR, Kurtin PJ, et al. A survey of clusterin and fascin expression in sarcomas and spindle cell neoplasms: strong clusterin immunostaining is highly specific for follicular dendritic cell tumor. Mod Pathol 2005;18:260-266.

28 Pillay K, Govender D, Chetty R. ALK protein expression in rhabdomyosarcomas. Histopathology 2002;41: 461-467.

29 Bridge JA, Kanamori M, Ma Z, et al. Fusion of the ALK gene to the clathrin heavy chain gene, CLTC, in inflammatory myofibroblastic tumor. Am J Pathol 2001;159:411-415.

30 Cools J, Wlodarska I, Somers R, et al. Identification of novel fusion partners of ALK, the anaplastic lymphoma kinase, in anaplastic large-cell lymphoma and inflammatory myofibroblastic tumor. Genes Chromosomes Cancer 2002;34:354-362.

31 Debelenko LV, Arthur DC, Pack SD, et al. Identification of CARS-ALK fusion in primary and metastatic lesions of an inflammatory myofibroblastic tumor. Lab Invest 2003;83:1255-1265.

32 Kazmierczak B, Cin PD, Sciot R, et al. Inflammatory myofibroblastic tumor with HMGIC rearrangement. Cancer Genet Cytogenet 1998;112:156-160.

33 Wlodarska I, De Wolf-Peeters C, Falini B, et al. The cryptic inv(2)(p23q35) defines a new molecular genetic 
subtype of ALK-positive anaplastic large-cell lymphoma. Blood 1998;92:2688-2695.

34 Yamamoto H, Oda Y, Saito T, et al. p53 Mutation and MDM2 amplification in inflammatory myofibroblastic tumours. Histopathology 2003;42:431-439.

35 Nonaka D, Birbe R, Rosai J. So-called inflammatory myofibroblastic tumour: a proliferative lesion of fibroblastic reticulum cells? Histopathology 2005;46: 604-613.
36 Lopez-Beltran A, Sauter G, Gasser T, et al. Infiltrating urothelial carcinoma. In: Eble JN, Sauter G, Epstein JI, et al (eds). World Health Organization Classification of Tumours: Tumours of the Urinary System and Male Genital Organs. IARC Press: Lyons, France, 2004, pp 93-109.

37 Cheville JC, Dundore PA, Nascimento AG, et al. Leiomyosarcoma of the prostate. Report of 23 cases. Cancer 1995;76:1422-1427. 